\title{
ESTUDO ELETROFISIOLÓGICO DO NERVO CUTÂNEO DORSAL LATERAL APLICABILIDADE TÉCNICA E VALORES DE REFERÊNCIA
}

RAFAEL JOSÉ SOARES DIAS*, ARMANDO PEREIRA CARNEIRO**

RESUMO - O estudo da condução neural dos segmentos mais distais dos nervos mais longos pode ser capaz de reconhecer mais precocemente as alterações oriundas da maioria das polineuropatias. O objetivo deste estudo, foi verificar a aplicabilidade técnica do exame de condução ortodrômica do ramo cutâneo dorsal do nervo sural (nervo cutâneo dorsal lateral) em pessoas saudáveis, padronizar os valores normais para serem utilizados como referência e comparar seus valores com os do nervo sural na perna. Quarenta e cinco pessoas com idade média de 41,56 anos (19-75) foram avaliadas, com registro de potenciais de ação de nervo sensitivo nos noventa pés. O eletrodo de captação foi colocado inferior e posteriormente ao maléolo lateral e a estimulação realizada $10 \mathrm{~cm}$ distalmente na face dorso-lateral dos pés. O valor médio para a velocidade de condução do nervo sural cutâneo dorsal encontrado foi 47,35 $\pm 4,8 \mathrm{~m} / \mathrm{s}$ e para a amplitude, $4,19 \pm 1,9 \mu \mathrm{V}$. A velocidade de condução do segmento distal foi $14 \%$ inferior à do proximal. A amplitude média dos potenciais de ação sensitivos do segmento distal foi $73 \%$ aquém daquela obtida no segmento proximal. Os resultados confirmam a possibilidade técnica de se estudar o ramo cutâneo dorsal do nervo sural e sugerem que o limite mínimo de normalidade para sua velocidade de condução, após correção para a temperatura de $34^{\circ} \mathrm{C}$, seja $38 \mathrm{~m} / \mathrm{s}$. Diferenças na amplitude e velocidade de condução devem ser consideradas entre grupos etários.

PALAVRAS-CHAVE: nervo sural, ramo cutâneo dorsal, condução sensitiva, condução neural , eletroneurografia.

Electrophysiological study of the lateral dorsal cutaneous nerve: thecnical applicability and normal values

ABSTRACT - The distal nerve conduction study of the long nerve in the leg is more efficient to work with so that it can stablish the early diagnosis of the majority of polyneuropathies. The main purpose of this study is the technical applicability of the orthodromic neural conduction examination of the dorsal cutaneous branch of the sural nerve (lateral dorsal cutaneous nerve) on healthy people, and define the normal values used as references to compare with the proximal segment. Forty five persons mean age 41.56 years old (range 19-75) were examined, and the sensory nerve action potentials were registered from ninety feet. The active recording superficial electrode was placed below and behind the lateral malleolus and the estimulating electrode was placed $10 \mathrm{~cm}$ distal to the recording superficial electrode at the dorsal lateral aspect of the feet. The mean value for the lateral dorsal cutaneous nerve conduction velocity was $47.35 \pm 4.8 \mathrm{~m} / \mathrm{s}$ and for amplitudes $4.19 \pm 1.9 \mu \mathrm{V}$. The sensory conduction velocity in the distal segment was $14 \%$ lower than the proximal one. The sensory nerve action potencial amplitude of the distal segment was $73 \%$ lower than the proximal one. The lower normal limit recomended for conduction velocity of this nerve plus correction for skin temperature of $34^{\circ} \mathrm{C}$ is $38 \mathrm{~m} / \mathrm{s}$. Some diferences in amplitude and conduction velocity among group ages are to be considered.

KEY WORDS: sural nerve, dorsal cutaneous branch, sensory nerve conduction, nerve conduction, electroneurography.

Estudo realizado na Clínica Eletroneurofisiatria Armando Carneiro, Belo Horizonte, MG: *Médico Fisiatra do Hospital Márcio Cunha, Ipatinga MG; **Médico Fisiatra e Neurofisiologista Clínico do Hospital Arapiara e da Clínica Eletroneurofisiatria Armando Carneiro. Aceite: 13-janeiro-2000.

Dr. Armando Pereira Carneiro - Av. do Contorno 2983 - 30110-080 Belo Horizonte MG - Brasil. Email:arpeiro@zaz.com.br 
O nervo sural é considerado um dos mais importantes no estudo eletrofisiológico para avaliação de neuropatias, pois é composto de fibras longas, encontra-se em posição anatômica superficial que permite fácil acesso e é relativamente livre de lesões por aprisionamento. Em neuropatias sistêmicas (polineuropatias), como na forma simétrica distal do diabete melito, as alterações do nervo sensitivo são consideradas mais precoces que as alterações da condução motora ${ }^{1}$. Entretanto, nem sempre nos deparamos com tal fato. Em recente estudo de 40 diabéticos, no qual foi realizado exame eletroneurográfico medindo a velocidade de condução dos nervos surais e tibiais, a alteração mais comum foi da velocidade de condução motora ${ }^{2}$.Diante desta situação, fica a possibilidade da alteração sensitiva do sural estar no segmento mais distal, uma vez que a velocidade foi avaliada entre a panturrilha e o tornozelo. O procedimento de se medir, por técnica antidrômica, a velocidade do nervo sural entre a perna e o tornozelo é o tradicionalmente utilizado ${ }^{3-5}$. A estimulação $14 \mathrm{~cm}$ acima do maléolo lateral, é comum nos laboratórios de neurofisiologia no Brasil e largamente divulgado pela literatura

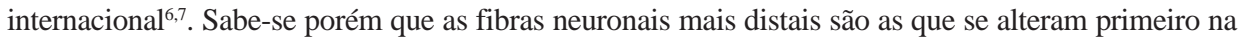
maioria das polineuropatias, no clássico padrão de degeneração axonal retrógada distal ("dying back").

O objetivo do presente estudo é verificar a possibilidade de se medir a condução do ramo cutâneo dorsal do nervo sural (nervo cutâneo dorsal lateral) nos pés de adultos saudáveis e obter valor padrão para determinação de casos patológicos. Os resultados são ainda comparados com os do nervo sural na perna.

\section{MÉTODO}

Avaliaramos 45 voluntários, a maioria funcionários do Hospital Arapiara. Foram 30 mulheres e 15 homens, com idade entre 19 e 75 anos (média 41,56 anos). Todos foram submetidos a anamnese e exame clínico para exclusão de trauma no tornozelo e presença de doença ou contato com substâncias tóxicas ou drogas lesivas ao sistema nervoso periférico. O estudo foi aprovado pelo Comitê de Ética Médica do Hospital e os voluntários assinaram declaração de consentimento.

O exame eletroneurográfico foi realizado com aparelho LBM série 4 (Neurodiagnostic Inc.), com eletrodo de captação do tipo barra. A condução foi feita ortodromicamente, com eletrodo de registro colocado em local anatomicamente definido como passagem do nervo sural ${ }^{8}, 1,4 \mathrm{~cm}$ inferior e posterior ao maléolo lateral da tíbia. A estimulação foi realizada $10 \mathrm{~cm}$ distal, na face lateral do dorso do pé, sendo o catodo posicionado no dorso (zona de transição da pele plantar com a dorsal) e o anodo na planta do pé (Fig 1). Foram avaliados os 2 pés de cada voluntário. $\mathrm{O}$ aparelho foi calibrado com $5 \mu \mathrm{V}$ por divisão com uma varredura de $10 \mathrm{~ms}$ ( $1 \mathrm{~ms} /$ divisão). Foi utilizado estímulo de $0,1 \mathrm{~ms}$ a 2 pulsos por segundo e promediação de 10 a 30 pulsos. A condução do sural na perna foi feita como anteriormante descrito ${ }^{9}$. A temperatura da pele foi medida no ponto médio entre o estímulo e a captação com termômetro digital modelo TM99A (Cooper Instrument Corp). As velocidades foram calculadas a partir da base da primeira deflecção negativa (latência de início) e os valores foram corrigidos para a temperatura de $34^{\circ} \mathrm{C}$ pela fórmula ${ }^{10}$ :

Velocidade corrigida $=$ valor medido $+(5 \%$ do valor medido $\mathrm{X} \Delta \mathrm{T})$.

$\Delta \mathrm{T}=34$ - temperatura medida

\section{RESULTADOS}

Os potenciais de ação sensitivos dos nervos cutâneos dorsais laterais foram obtidos em todos os 90 pés dos 45 voluntários avaliados. Os resultados dos valores médios e desvio padrão para as velocidades e amplitudes estão na Tabela 1 . Um exemplo da condução é mostrado na Figura 2. O valor médio das velocidades de condução dos surais nas pernas também se encontra na Tabela 1 . Comparativamente pudemos observar queda de $14 \%$ na velocidade de condução e de $73 \%$ na amplitude dos potenciais sensitivos do segmento distal em relação ao proximal.

Os valores das velocidades e amplitudes dos nervos cutâneos dorsais laterais por faixas etárias encontram-se na Tabela 2. Foi aplicado o teste F de análise de variância para comparação das médias das velocidades e amplitudes dos potenciais de ação sensitivos dos nervos cutâneos dorsais laterais por faixa etária. Os resultados demonstraram que, ao nível de significância de 5\%, as médias das 3 amostras não são iguais. O teste de Tukey demonstrou que a diferença estatisticamente significativa encontra-se entre as faixas etárias $19-45$ e a partir dos 60 anos. 


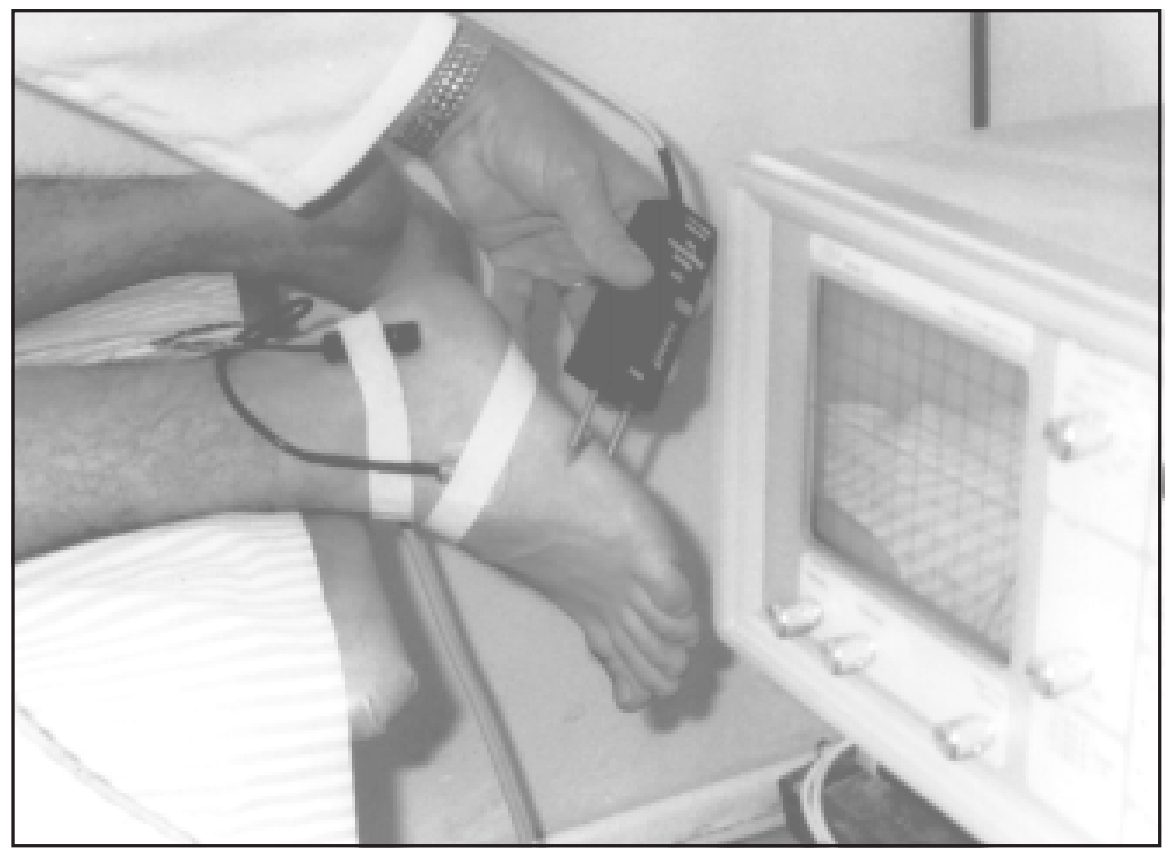

Fig 1. Colocação do eletrodo de captação e do estimulador para obtenção do potencial de ação de nervo sensitivo do nervo cutâneo dorsal lateral.

Tabela 1. Sumário dos achados eletrofisiológicos nos nervos sural e cutâneo dorsal lateral.

\begin{tabular}{lcccc}
\hline Nervo & Pés & V C* & A* & Idade** $^{*}$ \\
\hline Cutâneo dorsal lateral & 90 & $47,35 \pm 4,8(38-58)$ & $4,19 \pm 1,9(1,0-10)$ & $41,56(19-75)$ \\
Sural & 90 & $54,61 \pm 4,8(44-64)$ & $15,70 \pm 7,5(2,4-44)$ & $41,56(19-75)$ \\
\hline
\end{tabular}

VC, velocidade de condução; A, amplitude; * média, desvio padrão e variação; **média e variação.

Tabela 2. Achados eletrofisiológicos no nervo cutâneo dorsal lateral por faixa etária

\begin{tabular}{ccccc}
\hline Faixa etária & Pés & V C* & A & Idade *** \\
\hline 19 à 39 anos & 48 & $48,83 \pm 3,5(39-54)$ & $4,74 \pm 1,4(2,8-10)$ & $29,25(19-39)$ \\
40 à 59 anos & 22 & $46,05 \pm 5,4(38-57)$ & $4,56 \pm 2,4(1,4-9,2)$ & $46,64(40-58)$ \\
$\geq 60$ anos & 20 & $45,21 \pm 5,8(38-58)$ & $2,48 \pm 1,2(1,0-5,2)$ & $65,50(60-75)$ \\
\hline
\end{tabular}

VC, velocidade de condução; A, amplitude; * média, desvio padrão e variação; **média e variação.

\section{DISCUSSÃO}

Lee et al. ${ }^{11}$, em 1992, publicaram artigo que relata a utilização de estimulação antidrômica do nervo cutâneo dorsal lateral para medida da condução sensitiva. Em sua técnica, o eletrodo de captação é colocado na superfície dorso-lateral do pé, no ponto médio do $5^{\circ}$ metatarso e o estímulo realizado 


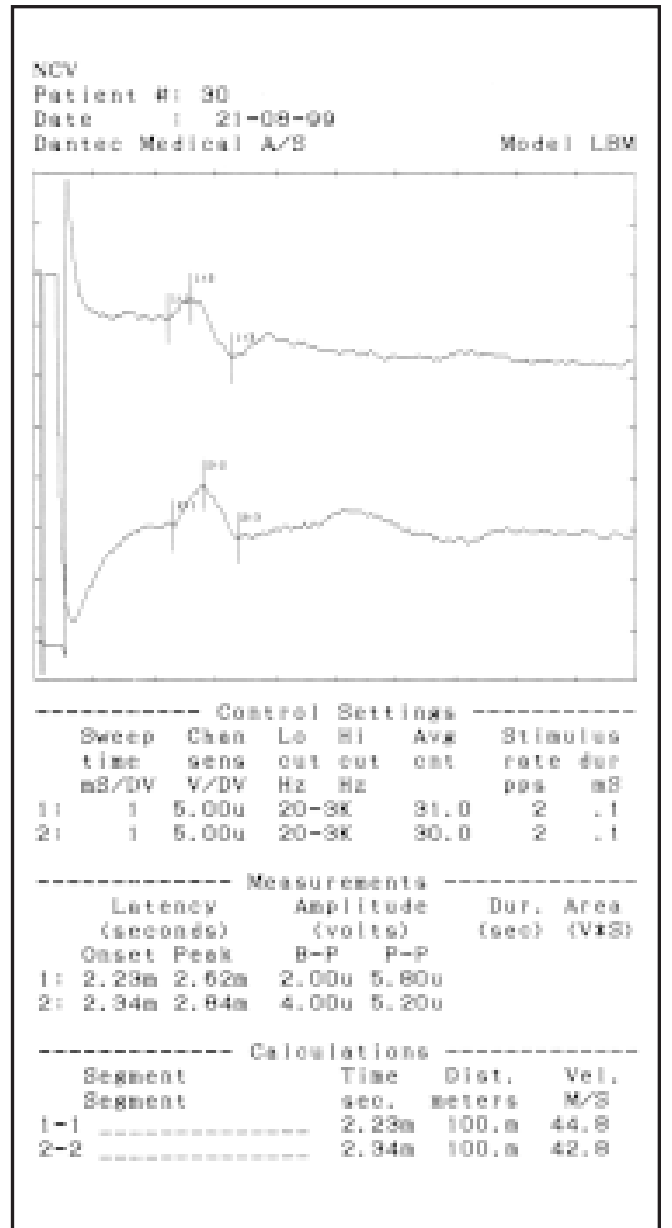

Fig 2. Potencial sensitivo do nervo cutâneo dorsal lateral.
$12 \mathrm{~cm}$ acima. Os resultados obtidos para as velocidades foram inferiores aos aqui relatados, sua média foi de $37,6 \mathrm{~m} / \mathrm{s}$. A diferença foi de $22 \%$. Tal fato, em parte, pode ser devido a temperatura, pois, enquanto no atual estudo ela foi corrigida para $34^{\circ} \mathrm{C}$, Lee et al. trabalharam com temperaturas a partir de $31^{\circ} \mathrm{C}$. Outros autores também têm utilizado temperaturas inferiores a $34^{\circ} \mathrm{C}$, para estudo da condução neural nos membros inferiores ${ }^{12}$. A opção pela correção para a temperatura de $34^{\circ} \mathrm{C}$ é devida à correlação temperatura da cutis/ músculo, em que a temperatura da pele de $34^{\circ} \mathrm{C}$ se associa a temperatura no músculo de $37^{\circ} \mathrm{C}^{13}$. O fator de correção utilizado para obtenção da velocidade teve como base os estudos de Johnson e Olsen ${ }^{10}$ que encontraram queda linear de $5 \%$ da velocidade por grau centígrado de variação da temperatura. Horowtiz e Krarup ${ }^{14}$ fizeram estudo ortodrômico da condução do nervo sural cutâneo dorsal captando com eletrodo de agulha justa neural no tornozelo, sendo os pés mantidos aquecidos a $35^{\circ} \mathrm{C}$. A média de velocidade obtida foi $46,2 \mathrm{~m} / \mathrm{s}$, bem semelhante à atual, com diferença de apenas $4 \%$. A proximidade dos resultados confirma o estudo de Franssen e Wienike ${ }^{15}$, de que o uso de fator de correção, ao invés de aquecimento, produz erro aceitável para as velocidades de condução neural, embora não ocorra o mesmo para outros parâmetros analisados (amplitude e duração). A velocidade média de condução do sural na perna na série de

Horowtiz e Krarup foi $55,5 \mathrm{~m} / \mathrm{s}$ e na de Grandini et al. ${ }^{12}$, foi 51,5, estes últimos porém obtiveram seus dados com temperatura cutânea de $29-30^{\circ} \mathrm{C}$. Isto posto, podemos considerar os resultados das 3 séries bastante semelhantes.

É oportuno considerar outras variáveis, além da temperatura, ao analisar a velocidade de condução neural, especialmente altura e idade. A altura tem relação inversa com a velocidade de condução neural, principalmente nos membros inferiores ${ }^{16,17}$. A idade também influencia negativamente tanto a velocidade quanto a amplitude das respostas sensitivas ${ }^{18,19}$, o que pode ser observado nos resultados por faixas etárias. É importante, ao comparar os resultados de pacientes com os limites do normal, ter em mente a influência dessas três variáveis, principalmente frente a resultados limítrofes.

Diante de tais fatos podemos concluir que o estudo da condução neural do nervo cutâneo dorsal lateral, através de técnica ortodrômica, é factível e fornece potenciais com amplitudes razoáveis, podendo ser utilizado nas avaliações de pacientes portadores de polineuropatias. Entretanto, a possibilidade de se diagnosticar polineuropatias precocemente com o estudo desse segmento ainda carece de demonstração. 
Neste estudo, nos preocupamos em estabelecer valores normais para velocidades e amplitudes, o estudo de patologias virá em seguida. $\mathrm{O}$ valor de referência para a velocidade de condução de adultos saudáveis foi $47,35 \mathrm{~m} / \mathrm{s} \pm 4,8$. Tomando - se como base 2 desvios padrões, o limite mínimo da velocidade de condução do ramo cutâneo dorsal do nervo sural foi $38 \mathrm{~m} / \mathrm{s}$, no presente estudo.

\section{REFERÊNCIAS}

1. Dumitru D. Electrodiagnostic medicine. Philadelphia. Hanley \& Belfus / Mosby, 1995:821-824.

2. Dias RJS, Vaz CJN, Jorge SMA, Macedo BD, Carneiro AP. Pé diabético: clínica, eletroneurografia e baropodometria. Medicina de Reabilitação 1999;51:11-16.

3. Schuchmann JA. Sural nerve conduction: a standardized technique. Arch Phys Med Rehabil 1977;58:166-168.

4. Truong XT, Russo FI, Vagi I, Rippel DV. Conduction velocity in the sural nerve. Arch Phys Med Rehabil 1979;60:304-308.

5. Kayed K, Røsjø Ø. Two-segment sural nerve conduction measurements in polyneuropathy. J Neurol Neurosurg Psychiatry 1983;46:867-870.

6. Johnson EW. Practical electromyography. 2Ed. Baltimore. Williams \& Wilkins, 1988:180-182.

7. Preston CD, Shapiro BE. Electromyography and neuromuscular disorders. Washington. Butterworth - Heinemann, 1998:134-135.

8. Lawrence SJ, Botte MJ. The sural nerve in the foot and ankle: an antomic study with clinical and surgical implications. Foot Ankle Int 1994:15:490-494.

9. Delisa JA, Lee HJ, Baran EM, Lai KS, Spielholz N. Manual of nerve conduction velocity and clinical neurophysiology. 3Ed.. New York. Raven Press, 1994: 144-145.

10. Johnson EW, Olsen kJ. Clinical value of motor nerve conduction velocity determinations. JAMA 1960;172:1- 6 .

11. Lee HJ, Bach JR, Delisa JA. Lateral dorsal cutaneous branch of the sural nerve. Am J Phys Med Rehabil 1992;71:318-320.

12. Grandini DL, Nóbrega JAM, Juliano Y. Valores normais das velocidades de condução nervosa em um grupo de 101 pessoas. Arq Neuropsiquiatr 1992;50:50-55.

13. Kimura J. Electrodiagnosis in diseases of nerve and muscle. 2Ed. Philadelphia. FA Davis, 1989:94-95.

14. Horowitz SH, Krarup C. Conduction studies of the normal sural nerve. Muscle Nerve 1992;15:374-383.

15. Franssen H, Wieneke GH. Nerve conduction and temperature: necessary warming time. Muscle Nerve 1994;17:336-334.

16. Rivner MH, Swift TR, Crout BA, Rhodes KP. Toward more rational nerve conduction interpretations: the effect of heigh. Muscle Nerve 1990;13:232-239.

17. Robinson LR, Rubner DE, Wahl PW, Fujimoto WY, Stolov WC. Influences of height and gender on normal nerve conduction studies. Arch Phys Med Rehabil 1993;74:1134-1138.

18. Stetson DS, Albers JW, Silverstein BA, Wolf RA. Effects of age, sex, and anthropometric factors on nerve conduction measures. Muscle Nerve 1992;15:1095-1104.

19. Falco FJE, Hennessey WJ, Goldberg G, Braddom RL. Standardized nerve conduction studies in the lower limb of the healthy elderly. Am J Phys Med Rehabil 1994;73:168-174. 